\title{
A Novel Entropy of Interval Valued Fuzzy Set
}

\author{
Chunfang Liu ${ }^{1,2, *}$ and YueSheng Luo ${ }^{1,3}$
}

${ }^{1}$ College of Automation, Harbin Engineering University, Harbin, Heilongjiang, 150001, P.R. China; ${ }^{2}$ College of Science, Northeast Forestry University, Harbin, Heilongjiang, 150001, P.R. China; ${ }^{3}$ College of Science, Harbin Engineering University, Harbin, Heilongjiang, 150001, P.R. China

\begin{abstract}
The main attribution of this paper is to propose two methods to construct entropy of interval valued fuzzy sets (IVFSs). First, we use an IVFS to construct two FSs and define entropy by similarity measure of the two FSs; then we use an IVFS to construct two IVFSs and also define entropy by similarity measure of the two IVFSs. At the same time, we give two examples to show that our methods are effective.
\end{abstract}

Keywords: Entropy, interval valued fuzzy set, similarity measure.

\section{INTRODUCTION}

Fuzzy sets (FS) was first introduced by Zadeh [1] which are used to deal with imprecision and uncertainty information. With the development of the fuzzy sets, many new theories came out, such as the interval valued fuzzy sets [2, $3,4]$ and the generalized theory of uncertainty [5]. Later Atanassov $[6,7]$ extended the fuzzy sets theory to the intuitionistic fuzzy sets (IFSs) which conclude membership function and non-membership function. Subsequently, some experts have applied the FSs and IVFSs theory to many fields. For example, Gorzalczany [8] researched the indicator of inclusion grade for IVFSs and applied to approximate reasoning, Turksen [9] studied the IVFSs in normal forms. Deschrijver [10] made some extentions of interval valued fuzzy sets. Moreover, Bustince [11] pointed out that intuitionistic fuzzy sets and interval valued fuzzy sets are equal in some degree.

It is an important topic to discuss the entropy and similarity measure in fuzzy sets theory. Entropy is a well-known concept in physics, information theory and fuzzy set theory. It describes the degree of uncertainties and fuzziness of the fuzzy sets. Since its appearance, some experts have made great progress in this field. For example, in 1972, in order to describe the fuzziness degree of fuzzy sets, De Luca and Termini [14] proposed some axioms of entropy. Burillo and Bustince [15] extended the definitions of entropy from FSs to the IFSs. Then, Szimidt and Kacprzyk [16] developed a new method to define entropy of IFSs using a geometric interpretation with nonprobabilistic-type. Based on distance, Zhang [17] proposed the new axiomatic definition of entropy for IVFSs and the relationship between entropy and similarity measure. The concept of similarity measure of fuzzy sets is to depict the similarity degree of fuzzy sets. It has many applications, such as fuzzy clustering, image processing, fuzzy reasoning and fuzzy neutral network $[5,13,17]$. Zeng and Guo [25] constructed similarity measure and entropy for IVFSs by normalized distances. Some researchers also have researched the similariy measure and entropy for IVFSs or IVIFSs [16-21] and have made great success in this field.

Consistently with the idea, we want to further study the entropy of IVFSs from the point of similarity measure. First, we use an IVFS to construct two FSs and define entropy by similarity measure of the two FSs; then we use an IVFS to construct two IVFSs and also define entropy by similarity measure of the two IVFSs. At the same time, we give two examples to show that our methods are effective.

The rest of our work is organized as follows. Section 2 gives basic definitions of the FSs and IVFSs. Section 3 constructs the new FSs and IVFS, respectively, and gives entropy of interval valued fuzzy sets. Section 4 gives our conclusion.

\section{PRELIMINARIES}

Definition 1 [1]. A fuzzy set $A$ in $X=\{x\}$ may be given as

$$
A=\left\{<x, u_{A}(x)>\mid x \in X\right\},
$$

where $u_{A}(x): X \rightarrow[0,1]$ is the membership function of $x$ in $A$.

Definition 2 [27]. We call a mapping $A: X \rightarrow[0,1]$ an interval valued fuzzy set in $X$. For every $A \in$ IVFSs and $x \in X, A(x)=\left[A^{-}(x), A^{+}(x)\right]$ is called the degree of membership of an element $x$ to $A$, then fuzzy sets $A^{-}: X \rightarrow[0,1]$ and $A^{+}: X \rightarrow[0,1]$ are called a low fuzzy set of $A$ and a upper fuzzy set of $A$, respectively. For simplicity, we denote $A=\left[A^{-}, A^{+}\right]$. 
Let $A, B$ be two IVFSs, then the following operations are obtained.

$$
\begin{aligned}
& A \subset B \text { iff } \forall x \in X, A^{-} \leq B^{-} \text {and } A^{+} \leq B^{+}, \\
& A=B \text { iff } \forall x \in X, A^{-}=B^{-} \text {and } A^{+}=B^{+}, \\
& A^{C}=\left[\left(A^{-}\right)^{C},\left(A^{+}\right)^{C}\right]=\left[1-A^{+}, 1-A^{-}\right]
\end{aligned}
$$

Definition 3 [15]. A real function $E:$ IVFSs $\rightarrow[0,1]$ is called an entropy on IVFSs, if $E$ satisfies the following properties:

(P1) $E(A)=0$ iff $A$ is a crisp set;

(P2) $E(A)=1$ iff $A^{-}+A^{+}=1$;

(P3) $E(A) \leq E(B)$ if $A$ is less fuzzy than $B$, i.e., $A^{-} \leq B^{-}$and $A^{+} \leq B^{+}$for $B^{-}+B^{+} \leq 1$ or $A^{-} \geq B^{-}$ and $A^{+} \geq B^{+}$for $B^{-}+B^{+} \geq 1$;

$$
\text { (P4) } E(A)=E\left(A^{C}\right)
$$

Definition 4 [23]. A real function $S:$ IVFSs $\times$ IVFSs $\rightarrow$ $[0,1]$ is called similarity measure of interval valued fuzzy sets, if $S$ satisfies the following properties:

(N1) $S\left(A, A^{C}\right)=0$ if $A$ is a crisp set;

(N2) $S(A, B)=1 \Leftrightarrow A=B$;

(N3) $S(A, B)=S(B, A)$;

(N4) for all $A, B, C \in$ IVFSs, if $A \subseteq B \subseteq C$, then

$$
S(A, C) \leq S(A, B), S(A, C) \leq S(B, C) .
$$

Specially, if interval valued fuzzy sets $A$ and $B$ become fuzzy sets, then $S(A, B)$ is similarity measure of fuzzy sets.

\section{ENTROPY OF INTERVAL VALUED FUZZY SET}

Let $A=\left[A^{-}, A^{+}\right]$be an IVFS, we construct two fuzzy sets $M^{-}, M^{+}$based on $A$.

$$
M^{-}=\frac{1+\left(A^{-}+A^{+}-1\right)^{2}}{2}, M^{+}=\frac{1-\left|A^{-}+A^{+}-1\right|}{2}
$$

Theorem 1. Suppose $S\left(M^{-}, M^{+}\right)$is the similarity of $M^{-}, M^{+}$, then $E(A)=S\left(M^{-}, M^{+}\right)$.

Proof. (P1) If $A$ is a crisp set, then for every $x \in X$, we have $A^{-}=A^{+}=1$ or $A^{-}=A^{+}=0$, it means that $A^{-}$ and $A^{+}$are crisp sets and $M^{-}=M^{+}=1$, therefore, $E(A)=S\left(M^{-},\left(M^{+}\right)^{C}\right)=0$.

(P2) Known by the definition of similarity measure of fuzzy sets, we have $E(A)=S\left(M^{-}, M^{+}\right)=1 \Leftrightarrow M^{-}=M^{+} \Leftrightarrow A^{-}+A^{+}=1$.

(P3) Since $A^{-} \leq B^{-}$and $A^{+} \leq B^{+}$with $B^{-}+B^{+} \leq 1$ implies that

$$
M_{A}^{-} \leq M_{B}^{-} \leq M_{B}^{+} \leq M_{A}^{+} .
$$

From the definition of similarity measure of fuzzy sets, we have

$$
S\left(M_{A}^{-}, M_{A}^{+}\right) \leq S\left(M_{A}^{-}, M_{B}^{+}\right) \leq S\left(M_{B}^{-}, M_{B}^{+}\right),
$$

then we successfully prove $E(A) \leq E(B)$.

With the same reason, when $A^{-} \geq B^{-}$and $A^{+} \geq B^{+}$ with $B^{-}+B^{+} \geq 1$, we can prove

$$
E(A) \leq E(B) \text {. }
$$

(P4) Because $A^{C}=\left[\left(A^{-}\right)^{C},\left(A^{+}\right)^{C}\right]=\left[1-A^{+}, 1-A^{-}\right]$, we have

$M_{A^{C}}^{-}=M^{-}, M_{A^{C}}^{+}=M^{+}$. From the definition of entropy and similarity measure of FSs, we get

$$
E(A)=S\left(M^{-}, M^{+}\right)=S\left(M^{+}, M^{-}\right)=S\left(M_{A^{C}}^{-}, M_{A^{C}}^{+}\right)=E\left(A^{C}\right)
$$

Hence, we complete the proof of Theorem 1.

Remark 1 We define entropy of IVFS A by similarity measure of two fuzzy sets $M^{-}, M^{+}$which are constructed by A. The construction is the extention of Zeng [26]. In Zeng's, the new FSs were $A^{-}, A^{+}$. While in ours, the new FSs are $M^{-}, M^{+}$which are the functions of $A^{-}, A^{+}$They both satisfy the definition of entropy of IVFSs.

Let $A=\left\{x \mid u_{A}(x)\right\}, B=\left\{x \mid u_{B}(x)\right\}$ be two FSs, and the Euclidean distance proposed by Deschrijver [10] is as follows:

$$
d(A, B)=\sqrt{\frac{1}{n} \sum_{i=1}^{n}\left|u_{A}\left(x_{i}\right)-u_{B}\left(x_{i}\right)\right|^{2}}
$$

and the similarity measure of two fuzzy sets $\mathrm{A} ; \mathrm{B}$ is as follows:

$$
S(A, B)=1-d(A, B)
$$

Example 1. Let $A=[0.2,0.4]$ and $B=[0.2,0.4]$ be two IVFSs, now we utilize (1), (2), (3) to obtain the entropy of $A, B$. 
For $A, M_{A}^{-}=0.58, M_{A}^{+}=0.3$ and $S\left(M_{A}^{-}, M_{A}^{+}\right)=0.72$, then we get $E(A)=0.72$.

For $B, M_{B}^{-}=0.52, M_{B}^{+}=0.4$ and $S\left(M_{A}^{-}, M_{A}^{+}\right)=0.88$, then we get $E(B)=0.88$.

We obtain $E(A)<E(B)$. For IVFSs, the longer the interval value is, the fuzzier the set is, so the result is consistent with our idea.

Next we develop another method to construct the entropy of interval valued fuzzy set which is on account of similarity measure of interval valued fuzzy sets.

For an IVFS $A$, for every $x$ in $X$, we construct two IVFSs $T_{A}, R_{A}$ and denote them $T_{A}=\left[T_{A}^{-}, T_{A}^{+}\right]$, $R_{A}=\left[R_{A}^{-}, R_{A}^{+}\right]$, where

$T_{A}^{-}=1-\left(\frac{A^{-}+A^{+}}{2}\right)^{2}, T_{A}^{+}=1-\frac{A^{-}+A^{+}}{2}$

$R_{A}^{-}=\left(\frac{A^{-}+A^{+}}{2}\right)^{2}, R_{A}^{+}=\frac{A^{-}+A^{+}}{2}$

Theorem2. Suppose $S\left(T_{A}, R_{A}\right)$ is the similarity of IVFSs $T_{A}, R_{A}$, and $E$ is entropy of IVFS, then $E(A)=S\left(T_{A}, R_{A}\right)$.

Proof. (P1) If $A$ is a crisp set, then for every $x \in X$, we have $A^{-}=A^{+}=0$ or $A^{-}=A^{+}=1$, it means that $T_{A}^{-}=T_{A}^{+}=1, R_{A}^{-}=R_{A}^{+}=0$ or $T_{A}^{-}=T_{A}^{+}=0, R_{A}^{-}=R_{A}^{+}=1$ we get $T_{A}=[1,1], R_{A}=[0,0]$ or $T_{A}=[0,0], R_{A}=[1,1]$, therefore, $E(A)=S\left(T_{A}, R_{A}\right)=0$.

(P2) Known by the definition of similarity measure of fuzzy sets, we have

$$
E(A)=S\left(T_{A}, R_{A}\right)=1 \Leftrightarrow T_{A}=R_{A} \Leftrightarrow A^{-}+A^{+}=1 .
$$

(P3) Since $A^{-} \leq B^{-}$and $A^{+} \leq B^{+}$with $B^{-}+B^{+} \leq 1$ which implies that

$$
R_{A} \subseteq R_{B} \subseteq T_{B} \subseteq T_{A} .
$$
have

From the definition of similarity measure of IVFSs, we

$$
S\left(T_{A}, R_{A}\right) \leq S\left(T_{B}, R_{A}\right) \leq S\left(T_{B}, R_{B}\right),
$$

then we successfully prove $E(A) \leq E(B)$.

With the same reason, when $A^{-} \geq B^{-}$and $A^{+} \geq B^{+}$ with $B^{-}+B^{+} \geq 1$, we can prove
$E(A) \leq E(B)$.

(P4) From the definition of $T_{A}, R_{A}$, we get

$T_{A}=T_{A^{C}}, R_{A}=R_{A^{c}}$ and $S\left(T_{A}, R_{A}\right)=S\left(T_{A^{C}}, R_{A^{C}}\right)$,

thus

$$
E(A)=E\left(A^{C}\right) \text {. }
$$

Hence, we complete the proof of Theorem 2.

Remark 2 The construction of the two IVFSs is also based on the IVFS $A$. In our construction, it is more simple in form and more easier to calculate than Zeng [23]. It provides a new method to calculate the entropy of IVFS.

Let $A=\left[A^{-}, A^{+}\right], B=\left[B^{-}, B^{+}\right]$be two IVFSs, and the Euclidean distance of two IVFSs proposed by Atanassov [7] is as follows:

$Q(A, B)=\sqrt{\frac{1}{2 n} \sum_{i=1}^{n}\left(A^{-}\left(x_{i}\right)-\left.B^{-}\left(x_{i}\right)\right|^{2}+\left|A^{+}\left(x_{i}\right)-B^{+}\left(x_{i}\right)\right|^{2}\right)}(6)$

and the similarity measure of two fuzzy sets $\mathrm{A} ; \mathrm{B}$ is as follows:

$S(A, B)=1-Q(A, B)$

Example 2. Let $A=[0.2,0.4]$ and $B=[0.2,0.4]$ be two IVFSs, now we utilize (4)-- (7) to obtain the entropy of $A, B$.

For $A, \quad T_{A}^{-}=0.91, T_{A}^{+}=0.7 \quad$ and $\quad R_{A}^{-}=0.09$, $R_{A}^{+}=0.3 \quad$ then we get $S\left(T_{A}, R_{A}\right)=0.2512$, $E(A)=0.2512$.

For $B, \quad T_{B}^{-}=0.84, T_{B}^{+}=0.6 \quad$ and $\quad R_{B}^{-}=0.16$, $R_{B}^{+}=0.4$, then we get

$$
S\left(T_{B}, R_{B}\right)=0.4988, E(B)=0.4988 .
$$

We obtain $E(A)<E(B)$, the result is consistent with example 1.

\section{CONCLUSION}

In this paper, we comment on the axiomatic definitions of entropy of IVFSs and proposed a new method to construct new FSs and IVFSs based on the IVFSs which are more simple in form and more easier to calculate. Furthermore, we obtained the entropy of IVFSs by the similarity measure of two FSs and IVFSs, repectively. Then we gave two examples to show that our methods are effective. The new methods developed and enriched the entropy of FSs and IVFSs.

\section{CONFLICT OF INTEREST}

The authors confirm that this article content has no conflict of interest. 


\section{ACKNOWLEDGEMENTS}

This work was financially supported by "the Fundamental Research Funds for the Central Universities" (2572014BB19).

\section{REFERENCES}

[1] L.A. Zadeh, Fuzzy sets, Inform. and Control 8 (1965) 338-353.

[2] L.A. Zadeh, The concept of a linguistic variable and its application to approximate reasoning (I), Information Science 8 (1975) 199249.

[3] L.A. Zadeh, The concept of a linguistic variable and its application to approximate reasoning (II), Information Science 8 (1975) 301357.

[4] L.A. Zadeh, The concept of a linguistic variable and its application to approximate reasoning (III), Information Science 9 (1975) 4380 .

[5] L.A. Zadeh, Toward a generalized theory of uncertainty (GTU)-an outline, Information Sciences 172 (2005) 1-40.

[6] K. Atanassov, Intuitionistic fuzzy sets, Fuzzy Sets and Systems 20 (1986) 87-96.

[7] K. Atanassov, Intuitionistic Fuzzy Sets: Theory and Applications, Physica-Verlag, Heidelberg, NewYork, 1999.

[8] H. Bustine, Indicator of inclusion grade for interval valued fuzzy set, Applications to approximate reasoning based on interval valued fuzzy sets, International Journal of Approximate Reasoning 23 (2000) 137-209.

[9] B. Turksen, Interval valued fuzzy sets based on normal forms, Fuzzy Sets and Systems 20 (1986) 191-210.

[10] G. Deschrijver, E. Kerre, On the relationship between some extensions of fuzzy set theory, Fuzzy Sets and Systems 133 (2003) 227235.

[11] H. Bustince, P. Burillo, Vague sets are intuitionistic fuzzy sets, Fuzzy Sets and Systems 79 (1996) 403-405.

[12] S.K. De.R.Biswas.A.R.Roy.Some operations on intuitionistic fuzzy sets.Fuzzy Sets and Systems114(2000)477-484.

[13] G.J. Wang, Y.Y. He, Intuitionistic fuzzy sets and L-fuzzy sets, Fuzzy Sets and Systems 110 (2000) 271-274.
[14] A.De Luca,S.Termini,A definition of a non-probabilistic entropy in the setting of fuzzy sets theory,Information and Control 20 (1972) 301-312.

[15] H. Bustince, P. Burillo, Entropy on intuitionistic fuzzy sets and on interval-valued fuzzy sets, Fuzzy sets and Systems 78 (1996) $305-$ 316.

[16] E. Szmidt, J. Kacprzyk, Entropy for intuitionistic fuzzy sets, Fuzzy Sets and Systems 118 (3) (2001) 467-477.

[17] H.Y. Zhang, W.X. Zhang, C.L. Mei, Entropy of interval-valued fuzzy sets based on distance and its relationship with similarity measure, Knowledge-Based Systems 22 (2009) 449-454.

[18] G.S. Huang, Y.S. Liu, The fuzzy entropy of vague sets based on non-fuzzy sets, Computer Applications and Software 22 (6) (2005) $16-17$.

[19] J.L. Fan, W.X. Xie, Distance measure and induced fuzzy entropy, Fuzzy Sets and Systems 133 (2003) 227-235.

[20] H.Y. Zhang, W.X. Zhang, C.I. Mei, Entropy of interval-valued fuzzy sets based on distance and its relationship with similarity measure, Knowledge Based Systems (2009) 449-454.

[21] W.L. Hung, M.S. Yang, Fuzzy entropy on intuitionistic fuzzy sets, International Journal of Intelligent System 21 (2006) 443-451.

[22] Y.C. Jiang, Y. Wang, Entropy on intuitionistic fuzzy soft sets and on interval-valued fuzzy soft sets, Information Science 240 (2013) 95-114.

[23] W.Y. Zeng, H.X. Li, Relationship between similarity measure and entropy of interval-valued fuzzy sets, Fuzzy Sets and Systems 157 (2006)1477-1484.

[24] X.C. Liu, Entropy, distance measure and similarity measure of fuzzy sets and their relations, Fuzzy Sets and Systems 52 (1992) 305-318.

[25] W.Y. Zeng, P. Guo, Normalized distance, similarity measure, inclusion measure and entropy of interval-valued fuzzy sets and their relationship, Information Sciences 178 (2008) 1334-1342.

[26] Z.S. Xu, Some similarity measures of intuitionistic fuzzy sets and their applications to multiple attribute decision making, Fuzzy Optimization and Decision Making 6 (2007) 109-121.

[27] C. Wang, A.J. Qu, Entropy, similarity measure and distance measure of vague soft sets and their relations, Information Sciences 244 (2013)92-106

(C) Liu and Luo; Licensee Bentham Open.

This is an open access article licensed under the terms of the (https://creativecommons.org/licenses/by/4.0/legalcode), which permits unrestricted, noncommercial use, distribution and reproduction in any medium, provided the work is properly cited. 